Rodas-Llanos, M. \& Cubero-Juánez, J. (2022). El diseño del artículo de investigación como generador de vocaciones científicas. Revista Electrónica Interuniversitaria de Formación del Profesorado, 25(1), 61-77.

\title{
El diseño del artículo de investigación como generador de vocaciones científicas
}

Manuel Rodas Llanos, Javier Cubero Juánez

Universidad de Extremadura, Facultad de Educación, Didáctica de las Ciencias

Experimentales y Matemáticas

\section{Resumen}

En este trabajo se describe una experiencia realizada con alumnos de enseñanza secundaria y bachillerato, en la que se plantea la elaboración de un artículo de investigación como recurso para generar actitudes positivas ante la ciencia y despertar vocaciones científicas. Se comprobó cómo el alumnado tenía escasos conocimientos acerca de la estructura de los artículos científicos, se estudiaron los diferentes modelos y se planteó la investigación utilizando como patrón la estructura de los mismos. Se emplearon antes y después de la experiencia dos cuestionarios, para evaluar el proceso y un tercero para testear las emociones generadas. El análisis del estudio mostró una disposición positiva del alumnado al aprendizaje de la ciencia mediante este método, porque entre otros aspectos les permitía profundizar en temas que consideraba de su interés.

\section{Palabras clave}

Aprendizaje activo; enseñanza secundaria; emociones; vocaciones científicas. 


\title{
The design of the research article as generator of scientific vocations
}

\begin{abstract}
This paper describes a didactic experience with secondary and high school students, in which it is proposed the elaboration of a research article as a resource to generate positive attitudes towards science and awaken scientific vocations. It was verified how the students had little knowledge about the structure of the scientific articles, the different models were studied and the research was considered using as a pattern the structure of the same. The process was evaluated by means of two questionnaires that were applied before and after the experience. A third questionnaire was used to test the emotions generated. The analysis of the study showed a positive disposition of the students to the learning of science by means of this method, because among other aspects it provided them the possibility of deepening in subjects that considered of their interest.
\end{abstract}

\section{Key words}

Activity learning; emotions; scientific vocation; secondary education.

\section{Introducción}

El mundo en el que vivimos, tiene cada vez más claro la necesidad que tiene de la Ciencia para su supervivencia, muy especialmente después de los grandes desafíos globales como el Calentamiento Global o la pandemia producida por el COVID-19. Parece imprescindible que las nuevas generaciones adquieran un conjunto de conceptos mínimos y aptitudes básicas en las disciplinas científicas. Autores como Buch, (2003) o Edwards et al., (2004) sugieren que esta formación científica de base, debe permitir a los jóvenes comprender y participar, con responsabilidad, en la búsqueda de soluciones a los problemas del mundo.

No obstante, y a pesar de la importancia que las administraciones educativas manifiestan por la adquisición de competencias tanto matemática como básicas en ciencia y tecnología, en la práctica, la prevalencia de un sistema anquilosado de enseñanza de la Ciencia, unido a currículos poco flexibles, ocasionan que en el aula no se potencie el empleo de procesos investigativos por parte del alumnado que les permitan desarrollar capacidades como la curiosidad, el rigor, el deseo de conocer, de plantearse preguntas, de observar, criticar, reflexionar y solucionar problemas. Este hecho, obstaculiza el desarrollo de competencias científicas en el estudiante que le permitan una aproximación cultural a la ciencia y la tecnología desde la escuela.

La VII Encuesta de Percepción Social de la Ciencia (FECYT 2017) recoge el interés espontáneo que se tiene por recibir noticias e informaciones sobre ciencia y tecnología. Al mismo tiempo, muestra cómo se produce un incremento del interés por la Ciencia en todas las franjas de edad, siendo la mayoritaria, la comprendida entre los 15 y 24 años, tendencia que se mantiene en la IX Encuesta de Percepción Social de la Ciencia 2018 (informe EPSCYT 2018), en la que se observa, además, una reducción progresiva de la brecha de género situándose en el 18,9\% entre los hombres frente al 13,9\% entre las mujeres. Así mismo, se advierte un mayor interés por la ciencia y la tecnología a medida que la edad de la persona entrevistada es menor, y cuanto mayor es el nivel de formación. 
A pesar de ello, hay que recordar, que el principal caballo de batalla de la ciencia es su divulgación, pues el motivo principal que se alega, para señalar el desinterés por la misma es la dificultad para entenderla. De ahí, que los principales medios que se empleen para "consumir" ciencias sean mayoritariamente la televisión e internet.

Pese a los esperanzadores datos sobre percepción social, se da la paradoja de que el alumnado, a la hora de elegir las materias que quiere cursar rehúye de las disciplinas científicas, como demuestran innumerables publicaciones. De hecho, las investigaciones realizadas sobre las actitudes mostradas por los alumnos hacia el aprendizaje de las ciencias arrojan dos claras conclusiones: por un lado se observa una creciente manifestación de actitudes negativas hacia las ciencias; por otro, se aprecian en función del género (Cheung, 2009; Vázquez \& Manassero, 2008) diferencias significativas en los posicionamientos del alumnado frente a las mismas. George, (2006) señala que la desafección es aún mayor entre las mujeres. Algunos autores sugieren que esa mayor aversión puede ser debida entre otras causas, al estrés o ansiedad que producen. Los estudios realizados por Udo (2004) y Bryant (2013) reportan que las chicas presentan mayores niveles de ansiedad que los chicos ante las ciencias.

Sin embargo, no solo el sexo parece ser el catalizador de estas diferencias, otros autores como Schreiner \& Sjøberg, (2004) concluyen que la disminución de las actitudes positivas hacia las ciencias aparece con mayor frecuencia en los países desarrollados y que además del género, aparecen otros factores que justifican tal comportamiento, como el nivel cultural, tipo de colegio, materia concreta, currículo y tipo de enseñanza que se imparta.

Parece sensato pensar que, si se potencian conductas negativas hacia las ciencias, se condicionará la elección de itinerarios en secundaria y bachillerato, lo que comprometerá futuros estudios universitarios relacionados.

Esto supone que como docentes somos responsables del desarrollo de las actitudes positivas (Cheung, 2009) por parte de nuestro alumnado, pero también, que debemos encontrar los motivos que les lleva a este rechazo.

Uno de ellos ya lo sugieren Pérez \& De Pro (2013) y Vázquez \& Manassero (2008), ya que destacan la poca relevancia que, en su opinión, tiene para sus vidas la ciencia e incluso lo aburrida que es.

En definitiva, la realidad que muestran los alumnos frente al aprendizaje de las Ciencias dista mucho de ser positiva, muy al contrario, en secundaria, muchos alumnos huyen de las materias científicas, lo que puede ser una causa más que esté provocando el descenso del número de estudiantes universitarios en las titulaciones de ciencias. Por si no fuera suficiente, están desapareciendo asignaturas relacionadas con la ciencia en el currículum oficial y muchas otras ven reducido sistemáticamente su carga lectiva, lo que agrava seriamente el problema.

Se hace pues preciso fomentar actitudes favorables en el alumnado hacia la Ciencia que favorezcan el proceso educativo, pues como apunta Hargreaves, (2000): "las emociones están en el corazón de la enseñanza" (p.558). También Mora, (2017) manifiesta: “La emoción, los sentimientos, sus mecanismos cerebrales y su expresión en la conducta siguen siendo la base, que debemos conocer para construir un edificio sólido en la enseñanza" (p.55). En ese mismo sentido García \& Sánchez, (2006) afirman que las actitudes del profesorado pueden favorecer o interferir directamente en el aprendizaje de los alumnos, por ello algunos autores plantean que las actitudes deberían ser consideradas como contenidos u objetivos que debieran trabajarse en la escuela (Pelcastre-Villafuerte et al., 2015). 
Sin embargo, como sugieren en su trabajo Mellado et al., (2014), las emociones no son fáciles ni de reconocer, ni de cuantificar, ni de clasificar, ni de evaluar por lo que se hace imprescindible la comprensión del dominio afectivo del alumno de ciencias, para inducir un cambio en las creencias y expectativas hacia las mismas, con actividades creativas y emocionantes que engendren actitudes positivas y por ende acerquen al alumno hacia las ciencias.

La producción de conocimiento es una de las ocupaciones principales de la Ciencia, se adquiere aplicando un método científico, es decir, un proceso estructurado, sistemático y autocorrectivo (Ramos-Álvarez \& Catena, 2004). Implica la generación de hipótesis originales acerca del objeto de estudio, la utilización de unos materiales y métodos adecuados para ponerla a prueba, el análisis e interpretación de los resultados obtenidos y finalmente su difusión entre la comunidad científica para que sea juzgado y si es pertinente incorporado al cuerpo del conocimiento científico.

En este contexto surge el artículo científico, que tiene como finalidad comunicar los resultados de investigaciones, ideas y debates de una manera clara, concisa y fidedigna, siendo la publicación por tanto uno de los métodos inherentes al trabajo científico, la cual es de suma importancia, ya que una investigación no se termina hasta que sus resultados sean publicados.

Pero es en este último paso, en la elaboración del artículo, donde el inv+stigador encuentra en no pocas ocasiones, las mayores dificultades (Slafer, 2009). Entonces ¿cómo elaborar un artículo? Camps, (2007) señala que todo artículo debe de presentar tres características básicas, debe ser ordenado, público y debe poder ser controlado por la comunidad científica.

El que un artículo sea ordenado implica que se emplee una estructura técnica determinada que sea conocida y aceptada por toda la comunidad. De hecho, aunque en un principio todas las publicaciones fueran de tipo descriptivo, pronto se hizo necesario describir en forma detallada la metodología empleada, surgiendo el sistema IMRYD, (figura 1).

,Cuâl es el problema?

Cómo se estudió el problema?

Qué se encontró?

Qué significan dichos hallazgos?
Introducción

Material y métodos

Resultados

Discusión

Figura 1. Formato IMRYD

\section{Objetivos}

A partir de esta problemática, el presente trabajo tiene como objetivo principal, que el alumno se sienta atraído por el estudio de las ciencias, para lo cual se plantea la elaboración de un artículo científico como expresión final del proceso de generación de Ciencia, que sea preciso, claro y conciso. Este proceso cumple a su vez con dos cometidos primordiales, favorecer el aprendizaje de la Ciencia y del método científico por un lado y fomentar la comunicación de lo estudiado por otro, lo cual como sugieren algunos autores puede beneficiar al curso de la propia investigación (Murillo et al., 2017).

Así mismo, se procura dentro del marco de adquisición de competencias científicas, que el alumno sea capaz de elaborar cuestionarios para la toma de datos y emplear mecanismos para su validación, que sea capaz de realizar el volcado de datos a una matriz de hoja de cálculo, de producir gráficos y aprender a interpretarlos, para la obtención de conclusiones. 
Finalmente, y dado que en la emoción se asientan los cimientos de la enseñanza, se pretende que el alumno muestre qué sensaciones ha tenido durante todo el proceso para compararlos con las emociones que sienten en las clases convencionales.

\section{Metodología}

\section{Muestra}

La experiencia se desarrolló durante el primer semestre del curso 2017/18, con un grupo de 46 alumnos y alumnas del IES Sierra de San Pedro en la Roca de la Sierra (Badajoz), pertenecientes a un grupo de $1^{\circ}$ de Bachillerato, formado por 18 chicas y 6 chicos que cursaban las asignaturas de Biología y/o Cultura Científica y otro grupo de $4^{\circ}$ de ESO de Biología y Geología, conformado por 12 chicas y 10 chicos. Es decir, casi dos tercios de la muestra son chicas $(n=30 ; 65,2 \%)$ y el resto chicos $(n=16 ; 34,8 \%)$. En cambio, por nivel $4^{\circ}$ ESO supone un $47,8 \%(n=22)$ del total muestral y $1^{\circ}$ bachillerato un $52,2 \%(n=24)$.

Puesto que la elección de asignaturas de ciencias en el sistema educativo es realizada en cuarto curso de la ESO, cabe señalar que todos los alumnos estudiados tenían a priori afinidad por las ciencias.

El trabajo se estructuró en dos fases, la experiencia propiamente dicha y su evaluación.

\section{Secuencia de Enseñanza}

La pauta empleada en la experiencia educativa fue la siguiente:

1. ¿Qué es un artículo de investigación? Se mostró a los alumnos los tipos de artículo que existen con ejemplos reales y sobre las temáticas que ellos mismos solicitaron.

2. Estructura típica del artículo. En segundo lugar, se les informó acerca de la estructura típica de un artículo, que sería la base de su trabajo. (Título, Autores, abstract, ...)

3. Título e introducción. A continuación, se le pidió al alumnado que trataran de proporcionar un título breve, sugerente y lo más explicativo posible para su futuro artículo. Posteriormente se realizaron búsquedas de información en la red, partiendo de la idea principal y/o el título propuesto. Aunque se les informó de la existencia de algunas bases de datos importantes, se empleó el buscador académico de google, para localizar artículos relacionados con su estudio.

4. Materiales y métodos. En primer lugar, se procedió a la elaboración y validación de entrevistas y cuestionarios con Inflesz 1.o, seguidamente a la decisión de la población objeto de estudio y finalmente a la obtención de datos y al volcado de los mismos a hoja de cálculo del programa, Libreoffice. Para los trabajos que implicaron un diseño experimental, se procedió a la modificación de estudios previos sobre el tema en cuestión.

5. Análisis de los datos obtenidos y conclusiones. Se basó en la producción e interpretación de gráficos y el desarrollo de conclusiones.

6. Realización del resumen, palabras clave y bibliografía.

7. Revisión del artículo. Reformulación del título.

8. Presentación de los trabajos. Como resultado final del proceso se debían comunicar a la comunidad científica las investigaciones realizadas. Se elaboraron pósteres para su exposición, durante la semana de la Ciencia y finalmente en las reuniones científicas para alumnos de secundaria. 


\section{Fase de Evaluación}

La evaluación se fundamentó además de en la observación directa del proceso y la obtención de los resultados finales en forma de artículo, en dos cuestionarios de preguntas abiertas validados con inflesz 1.0 y por expertos de las áreas de Didáctica de las Ciencias Experimentales y Psicología de la UEX. Para comprobar su pertinencia fueron además probados, con otro grupo de $4^{\circ}$ de ESO que sirvió de blanco.

Estos cuestionarios fueron completados por el alumnado antes de la experiencia (cuestionario inicial) y al cabo de un mes de su finalización (cuestionario final).

Para observar las emociones que había desarrollado el alumnado durante el proceso, se administró un tercer cuestionario como instrumento, que consistía en una escala tipo Likert con 10 ítems y 5 posibles respuestas, constituida por díadas de emociones opuestas, tales como motivación-desaliento o entretenimiento-aburrimiento, adaptada de García-Ruiz y Sánchez (2006) y que para facilitar su análisis posterior fue reducido a solo 3. De tal forma, que a los dos grados que reflejaban la emoción positiva se les otorgó el valor 1; el valor 2 al grado que indicaba la neutralidad y el valor 3 para los dos grados que expresaban el sentimiento negativo frente a las emociones analizadas.

Este cuestionario se aplicó en dos momentos diferentes. Coincidiendo con el cuestionario inicial se preguntó al alumnado acerca de las emociones que les producía la enseñanza tradicional las Ciencias y antes de finalizar el proceso, se volvió a preguntar por las emociones que habían sentido, pero con el método de enseñanza objeto de estudio.

\section{Análisis de Datos}

El cuestionario inicial arrojó los siguientes datos: Índice Flesch-Szigriszt 69,42; Grado en la escala Inflesz: Bastante Fácil e Índice Fernández Huerta 74,27.

El cuestionario final presentó un Índice Flesch-Szigriszt de 72,84; Grado en la escala Inflesz: Bastante Fácil e Índice Fernández Huerta de 77,38, lo que indica su sencillez de comprensión.

El Cuestionario de emociones adaptado, mostró una fiabilidad muy aceptable al presentar un alfa de Cronbach de 0,84.

Para conocer las diferencias de percepciones que presentaba en función del género y nivel educativo, se aplicó la prueba U-Mann-Whitney para muestras independientes, dado que la muestra no se ajustaba a una distribución normal, (Shapiro-Wilk $\mathrm{p}<0,05$ ). Por otro lado, para estudiar las emociones que manifiesta el alumno tanto para la enseñanza tradicional como para el sistema de aprendizaje en estudio, se empleó la prueba de los Rangos de Wilcoxon.

\section{Resultados y Discusión}

\section{Cuestionario Inicial}

El análisis del mismo mostró que más del $65 \%$ del alumnado es conocedor del método científico, aunque ninguno de los encuestados señaló que para alcanzar el fin último de la creación de conocimiento era necesario dar a conocer lo hallado.

Por otro lado, si se les interrogaba acerca de cuál eran las fases habituales de un trabajo científico, las contestaciones eran muy dispares, las cuales fueron clasificadas y codificadas como se puede observar en la tabla 1. 
Tabla 1.

¿Cuáles crees que son las fases habituales de un trabajo científico?

\begin{tabular}{|c|c|c|c|c|c|c|c|}
\hline Tipo de Respuesta & Ejemplo de & Alt & Imnos & Alu & mnas & & tales \\
\hline $\begin{array}{l}\text { Utilizando método } \\
\text { científico }\end{array}$ & $\begin{array}{l}\text { "Siguiendo el } \\
\text { método científico" }\end{array}$ & 4 & $25,00 \%$ & 9 & $30,00 \%$ & 13 & $28,26 \%$ \\
\hline $\begin{array}{l}\text { Respuestas relacionadas } \\
\text { con el estudio }\end{array}$ & $\begin{array}{l}\text { “Exposición clara del } \\
\text { problema en el aula" }\end{array}$ & 3 & $18,75 \%$ & 12 & $40,00 \%$ & 15 & $32,61 \%$ \\
\hline $\begin{array}{l}\text { Respuestas relacionadas } \\
\text { con el concepto de } \\
\text { trabajo }\end{array}$ & $\begin{array}{l}\text { "Hacer las cosas con } \\
\text { limpieza, precisión y } \\
\text { orden” }\end{array}$ & 4 & $25,00 \%$ & 3 & $10,00 \%$ & 7 & $15,22 \%$ \\
\hline $\begin{array}{l}\text { Respuestas relacionadas } \\
\text { con la utilidad/interés del } \\
\text { trabajo }\end{array}$ & $\begin{array}{l}\text { "Para obtener la } \\
\text { tesis doctoral" }\end{array}$ & 2 & $12,50 \%$ & 0 & $0,00 \%$ & 2 & $4,35 \%$ \\
\hline No sabe/no contesta & & 3 & $18,75 \%$ & 6 & $20,00 \%$ & 9 & $19,57 \%$ \\
\hline
\end{tabular}

De dicha tabla, se puede inferir que alrededor de uno de cuatro alumnos independientemente del sexo seguirían el método científico. No obstante, lo que verdaderamente llama la atención es que alrededor del $40 \%$ de las chicas creen que las fases de una investigación están más bien relacionadas con un estudio del tema y su posterior exposición a los demás.

Así mismo, el cuestionario puso de manifiesto (figura 2) que dos de cada tres alumnos afirmaban que habían leído alguna vez un artículo científico, siendo las principales temáticas las relacionadas con la Química, Astronomía, Biología y Medicina. No obstante, como era previsible, existe una diferencia significativa entre alumnos de $4^{\circ}$ de la ESO y de $1^{\circ}$ bachillerato, constatándose que mientras que sólo un $50 \%$ de los alumnos de $4^{\circ}$ de la ESO afirman haber leído algún artículo en bachillerato lo hacen el $87,5 \%$. Como peculiaridad cabe reseñar que mientras que en bachillerato no existen diferencias por sexo, en $4^{\circ}$ son mayoritarios los varones. Esta particularidad coincide con lo apreciado por autores como George, (2006) o Vázquez y Manassero (2008), pues en $4^{\circ}$ de ESO la división entre Ciencias Naturales y Ciencias Sociales no es tan notable como en bachillerato, donde el alumnado ha decidido claramente por las primeras y por tanto, se presupone, que su actitud hacia las mismas debe ser positiva y mostrar un mayor interés.

En cuanto al objetivo de un trabajo científico, aparecen discrepancias entre alumnos de $4^{\circ}$ de ESO y de $1^{\circ}$ de Bachillerato, mientras que en este último casi el $50 \%$ señala como objetivo fundamental el aprendizaje y el 31\% la información, para los estudiantes de $4^{\circ}$ aunque no hay un objetivo claro señalan como principal la investigación con el 32\%. 
Sin embargo, si se les preguntaba cuál era su utilidad, más del $85 \%$ manifestaban que no la conocían, mientras que el resto afirmaban que era para investigar o informar sobre algún tema.

Figura 2.

Alumnos que manifiestan que han leído algún artículo científico en las asignaturas de ciencias (en claro), frente a los que señalan (en oscuro) que no.

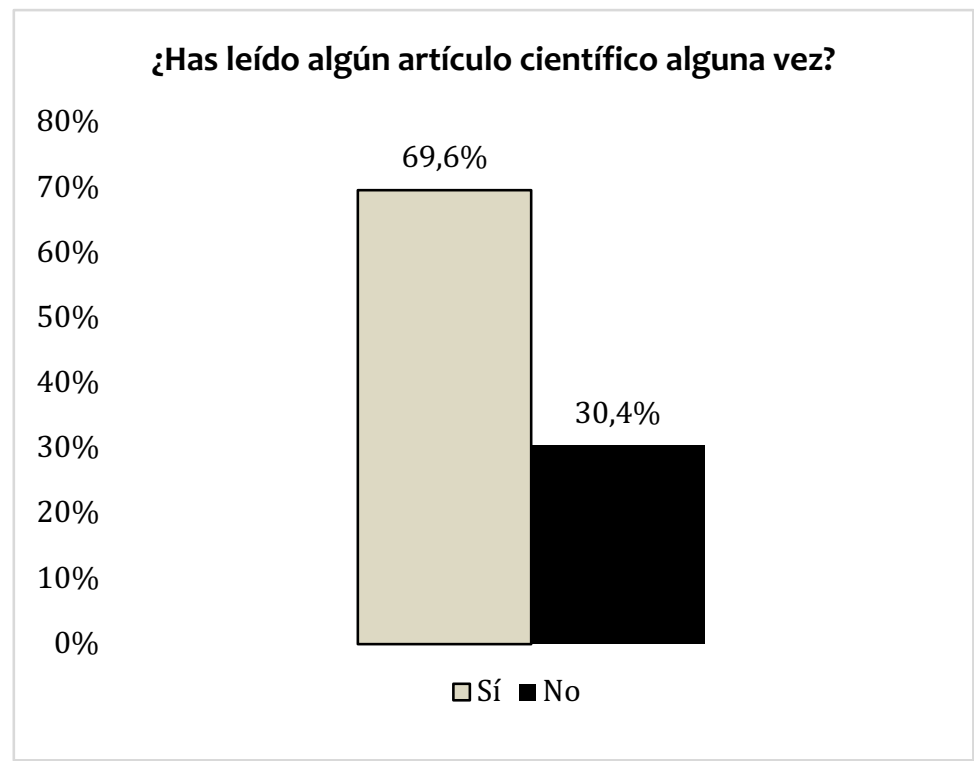

\section{Experiencia Educativa}

La producción investigadora finalmente obtenida, como consecuencia de la experiencia fue la siguiente:

Se seleccionaron 8 artículos, 6 que implicaban nuevo conocimiento y otros dos que suponían artículos de revisión debido a la especial dificultad de los mismos. Este hecho, se puso de manifiesto en la obtención de información, así, por ejemplo, la búsqueda del vocablo “distroglicanopatía" con Google Académico arrojó solo 8 resultados. Los títulos de los mencionados artículos fueron:

- Estudio de los caracteres poligénicos presentes en los alumnos del IES Sierra de San Pedro.

- Las plantas marcan el ritmo.

- "El saber pesa", pero la mochila más.

- ¿Perro peligroso?

- Geminando en clave de Sol

- El móvil en adolescentes.

- Huntington y la Proteína Errónea.

- Enfermedades asociadas al distroglicano 
Mencionar que, dentro del proceso metodológico, los diferentes grupos seleccionaron diferentes segmentos de población y estrategias en función de sus intereses. Así, por ejemplo, mientras el estudio de los caracteres poligénicos se hizo a todos los alumnos del Centro empleando un cuestionario de salud, el estudio sobre las razas de perros peligrosos se centró en profesores, PAS y AMPA del IES que tuvieran mascotas, a través de entrevistas personales, como se puede comprobar en la tabla 2.

Tabla 2.

Ejemplos de preguntas elaboradas por los alumnos para las entrevistas y cuestionarios.

\begin{tabular}{|c|c|}
\hline \multicolumn{2}{|c|}{ Ejemplo de entrevista sobre perros peligrosos } \\
\hline \multicolumn{2}{|c|}{$\mathrm{P}$ ¿A qué crees que se debe que un perro tenga un mal comportamiento? } \\
\hline \multicolumn{2}{|c|}{$\mathrm{R}$ A que no se le ha educado convenientemente } \\
\hline \multicolumn{2}{|c|}{ P Tendrías un perro de raza considerada "peligrosa" si tienes niños pequeños } \\
\hline \multicolumn{2}{|l|}{ R De ningún modo. } \\
\hline \multicolumn{2}{|c|}{ Ejemplo de cuestionario con respuesta tipo test } \\
\hline \multicolumn{2}{|c|}{ 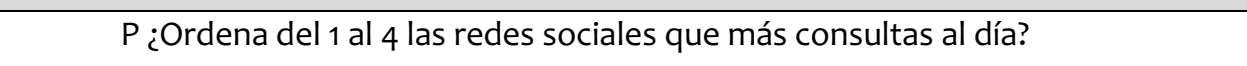 } \\
\hline - WhatsApp & - $\quad$ Skype \\
\hline - $\quad$ Twitter & - $\quad$ Gmail \\
\hline - $\quad$ Facebook & - $\quad$ Google + \\
\hline - Instagram & - Snapchat \\
\hline
\end{tabular}

Otro caso diferente, fue el estudio sobre germinación y crecimiento de leguminosas sometidas a diferentes estilos musicales, en el que los alumnos debieron diseñar el proceso experimental en laboratorio, empleando el método heurístico denominado "de prueba y error".

El alumnado recogió sus resultados en la hoja de cálculo y elaboró gráficas como la que se recoge en la figura 3 referente al color del pelo.

Del mismo modo elaboraron sus conclusiones incluso referenciadas, como en el caso de la revisión sobre el mal de Huntington que se recoge en la figura 4.

Una vez realizados los artículos científicos, se le planteó al alumnado la posibilidad de presentar una comunicación de su trabajo a un congreso. Se seleccionó el formato póster y se enviaron a la XXII Reunión Científica para estudiantes de Secundaria 2018 (figura 5). Por otro lado, también se planteó el envío de uno de los artículos, "Las plantas marcan el ritmo", a la revista de investigación científica para alumnos de secundaria, Meridies. 
Figura 3.

Gráfica elaborada por los alumnos para el carácter "color de pelo"

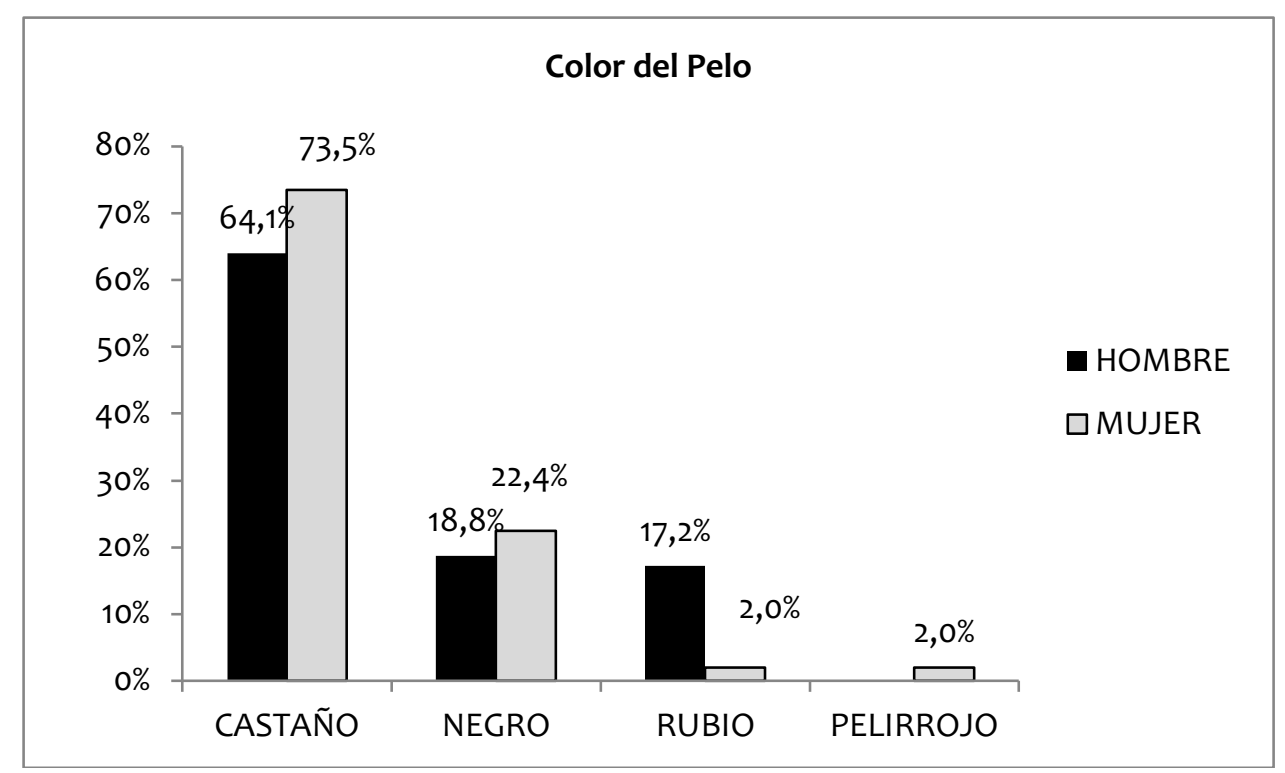

Figura 4.

Ejemplo de conclusiones referenciadas empleadas por los alumnos

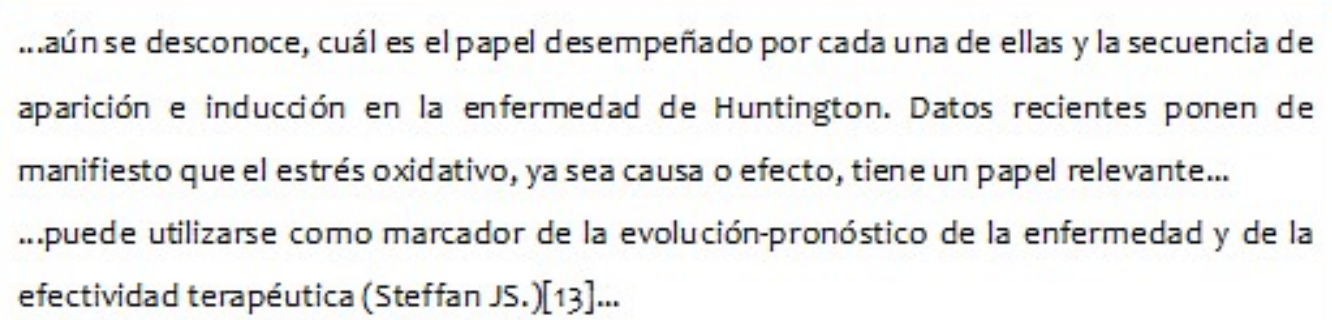

Figura 5 .

Pósteres elaborados para la XXII Reunión Científica.
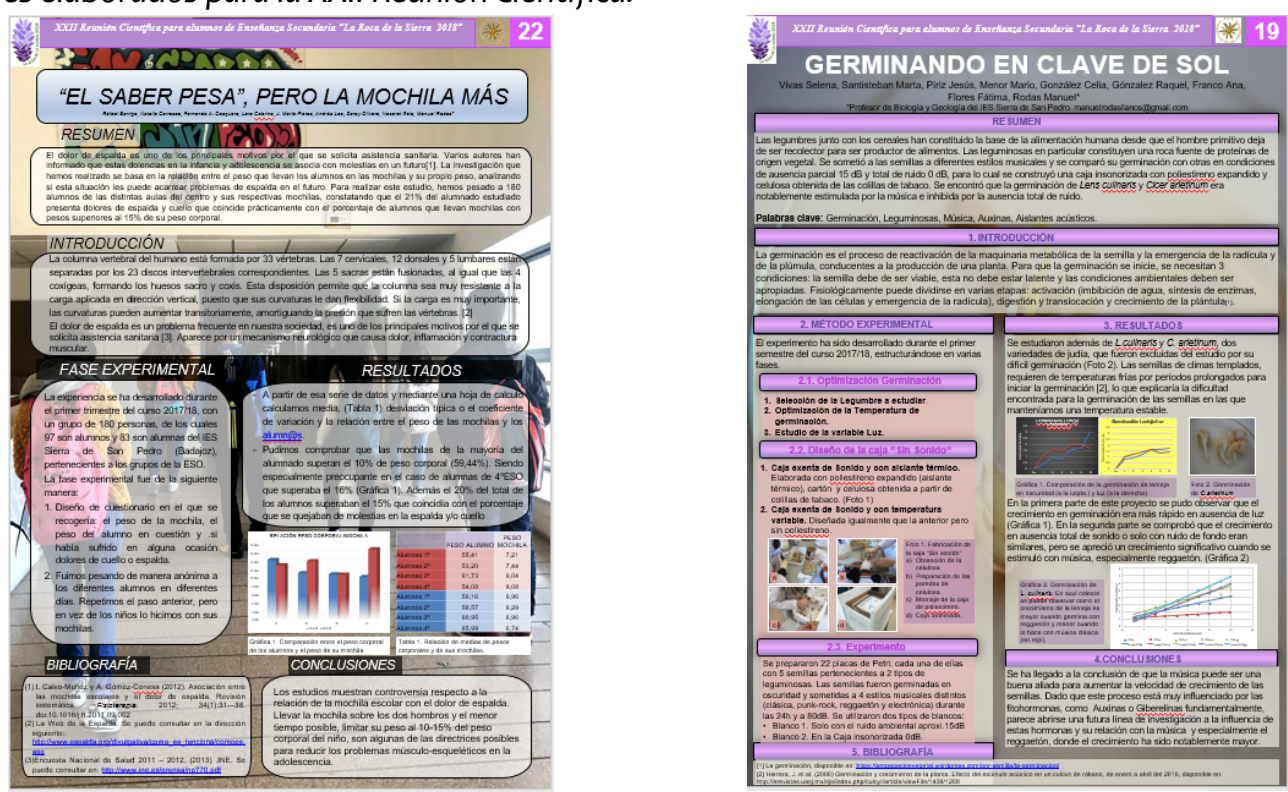


\section{Cuestionario Final}

Los datos recogidos indican que el alumnado, señala la necesidad de publicar los datos obtenidos en las investigaciones, bien a través de un artículo o de otro medio, con el fin de avanzar en la Ciencia. Del mismo modo, se aprecia una mejora significativa en el conocimiento de la estructura del artículo de investigación, pues a la pregunta formulada en el primer cuestionario "enumera las partes de un artículo", el 83,3\% ya reconoce varias partes de un artículo científico y el $16,7 \%$ las reconoce todas, frente al $83,9 \%$ que en el primer cuestionario no conocía ninguna (figura 6).

Figura 6.

Evaluación del aprendizaje del alumno antes (negro) de haber realizado la experiencia y al mes después de la misma (claro).

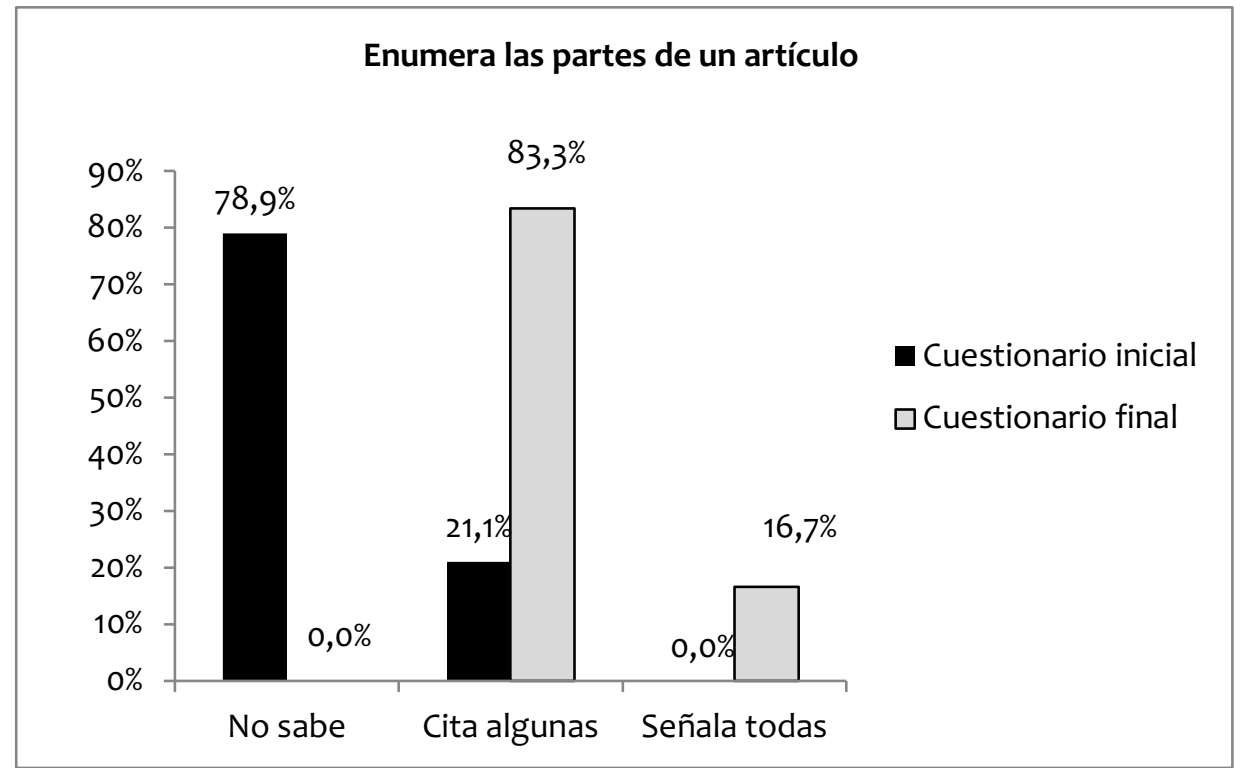

Como era de esperar, el porcentaje de alumnos que indica que ha leído una artículo científico aumenta del $67,4 \%$ en el primer cuestionario al $86,7 \%$ en el segundo; sin embargo se aprecia una variación significativa en la opinión que el alumnado ha adquirido para el concepto de artículo de investigación, pues si en principio el $71 \%$ del alumnado afirmaba que había empleado el artículo de investigación en otras asignaturas, fundamentalmente Música, Filosofía o Ciencias Sociales, ahora se produce un sorprendente vuelco de la situación pues el $80 \%$ afirma que solo han trabajado algún artículo de prensa, pero que no los pueden considerar de investigación en ningún caso (figura 7).

Así mismo, cuando al alumno se le pide que indique dónde ha encontrado mayores dificultades para realizar su trabajo, señala cuatro factores: en primer lugar la búsqueda de referencias para elaborar una buena introducción y diseñar sus experimentos, en segundo lugar la redacción en sí del artículo, puesto que no están acostumbrados a ajustarse a unos parámetros previamente establecidos, en tercer lugar, todo el procedimiento experimental, pues señalan que les ha obligado a plantear diferentes vías para resolver las situaciones problemáticas que se les planteaban y finalmente el análisis de los datos obtenidos. 
Figura 6.

Evolución de la opinión del alumnado para el concepto de artículo de investigación. En claro, alumnos que han leído artículos en asignaturas que no son de Ciencias, en negro los que no.

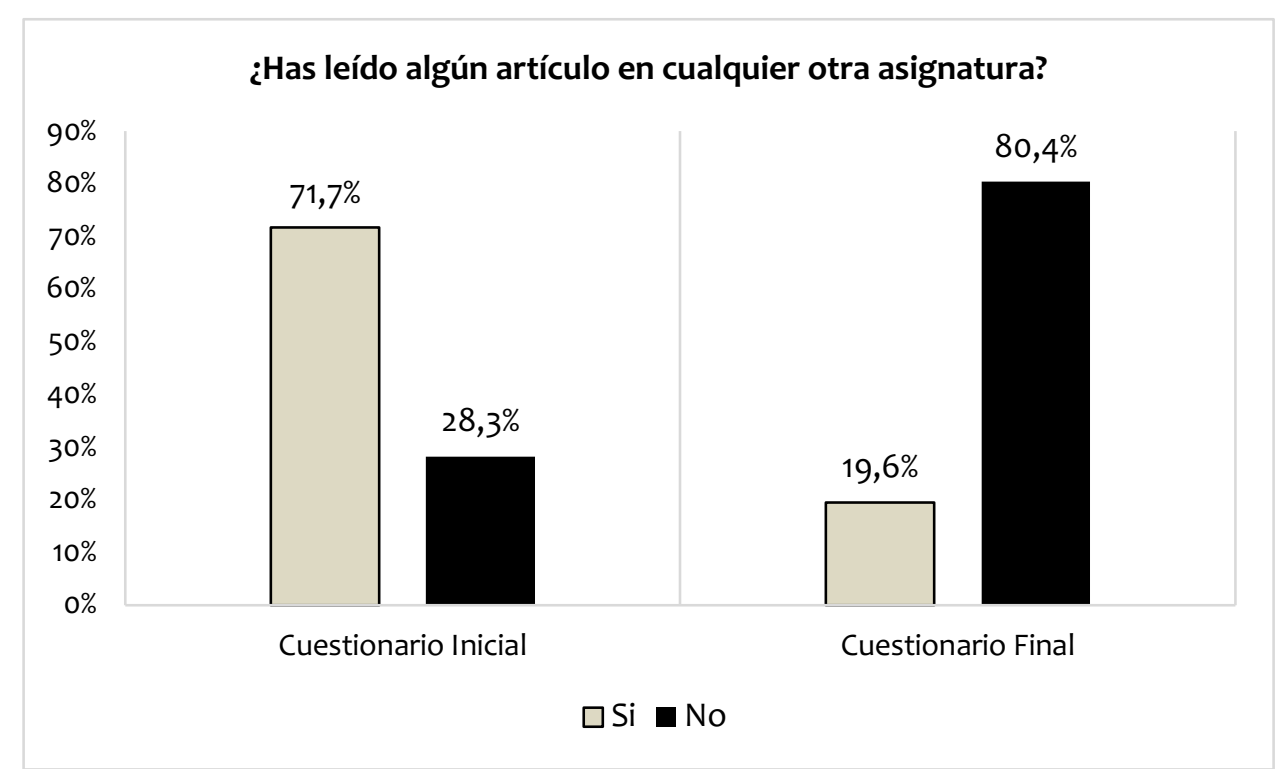

En cuanto a la opinión que le merecía el sistema de aprendizaje, la mayoría lo califican como interesante, poco común, original y divertido, que evita el tedio de las clases tradicionales y que por tanto es una manera creativa de aprender. Dado lo cual las emociones que manifiestan son en general positivas, de hecho, el $67 \%$ del alumnado reseñó su satisfacción por el trabajo, debido fundamentalmente a que el proceso les había resultado más sencillo de lo que a priori esperaban, porque habían sentido que realmente estaban aprendiendo algo y porque el tema que trataban era interesante y por tanto útil. Por contra el 33\% restante manifestó que le había supuesto mucho trabajo y que le había sido difícil. A pesar de este último mal dato, el 95,7\% de los estudiantes revelan que les gustaría repetir el proceso.

\section{Cuestionario de Emociones}

De las emociones estudiadas encontramos varios datos significativos que se pueden apreciar en la tabla siguiente (Tabla 3):

Tabla 3.

Prueba de los Rangos de Wilcoxon para las díadas de emociones.

\begin{tabular}{lcccc}
\hline Díadas & $\begin{array}{c}\text { Entretenida- } \\
\text { Aburrida }\end{array}$ & $\begin{array}{c}\text { Atracción- } \\
\text { Rechazo }\end{array}$ & $\begin{array}{c}\text { Claridad- } \\
\text { Confusión }\end{array}$ & $\begin{array}{c}\text { Tranquilidad- } \\
\text { Tensión }\end{array}$ \\
\hline Z & $-3,614^{\mathrm{a}}$ & $-2,677^{\mathrm{a}}$ & $-1,995^{\mathrm{b}}$ & $-2,064^{\mathrm{b}}$ \\
\hline Sig. asintótica (bilateral) & 0,000 & 0,007 & 0,046 & 0,039 \\
\hline
\end{tabular}

a. Basado en rangos positivos.

b. En rangos negativos.

Como se puede comprobar (tabla 3), la enseñanza tradicional es considerada más aburrida $(p=0,000)$ y es menos atractiva $(p=0,007)$ que la empleada en este estudio (figura 8). Por el contrario, el alumno considera que este tipo de enseñanza basado en la producción de un 
artículo, genera más confusión $(\mathrm{p}=0,046)$ y provoca más tensión $(\mathrm{p}=0,039)$ que la enseñanza tradicional. Preguntados posteriormente los alumnos acerca de este pormenor, contestaron que era debido a la inseguridad que les generaba el no saber qué hacer en algunos momentos.

En los cuadros superiores de la figura 8, se puede comprobar como la enseñanza experimental es percibida como más entretenida (izquierda) y genera mayor atracción (derecha) que la tradicional. En los inferiores, por el contrario, se pone de manifiesto que la enseñanza tradicional genera más claridad (izquierda) y produce una mayor sensación de tranquilidad (derecha) que la experimental.

Figura 7.

Díadas de emociones.
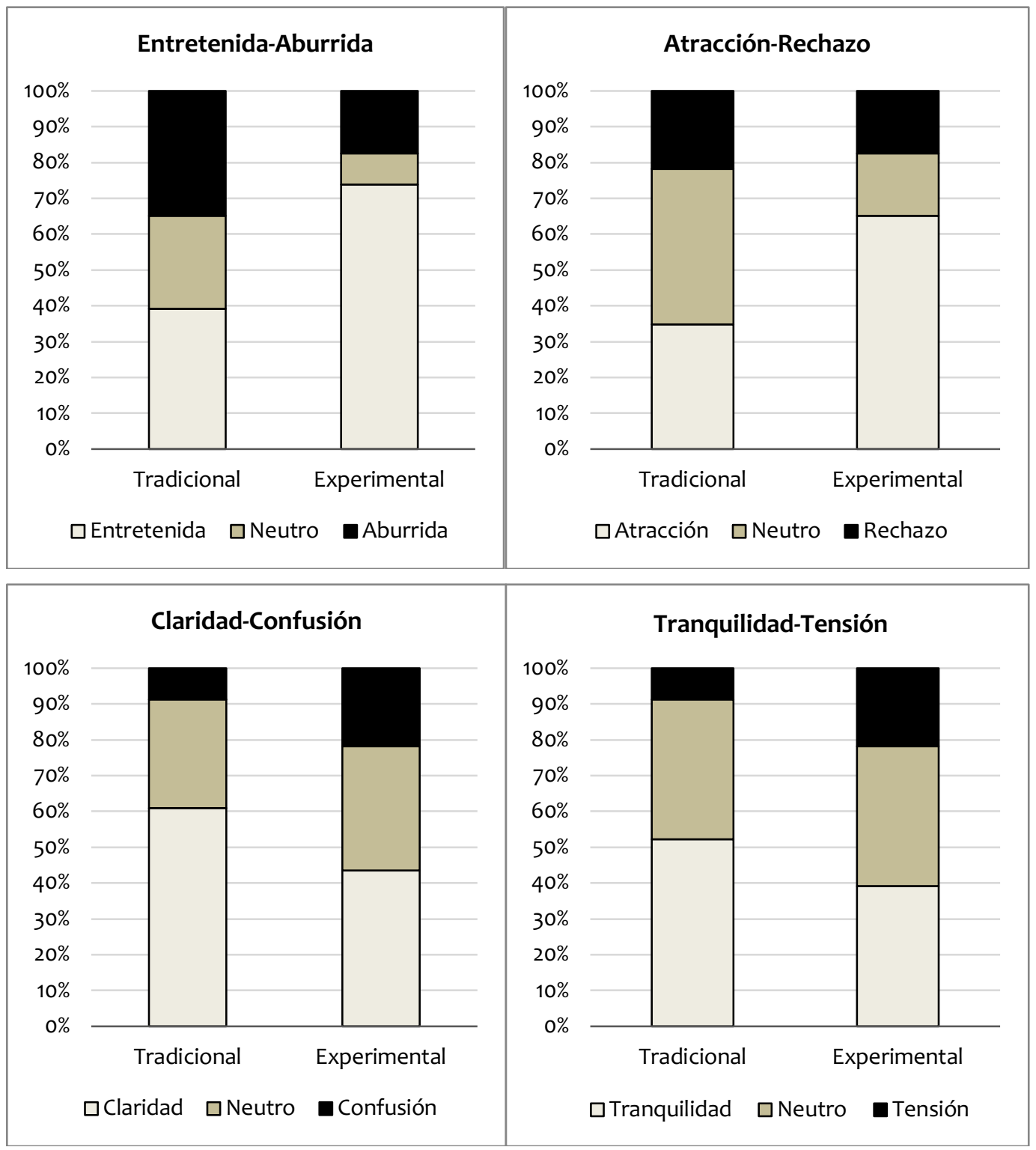

Se testeó si existían diferencias en las emociones que expresaban chicos y chicas y para ello, se empleó la prueba $U$ de Mann-Whitney. 
En la tabla 4 se aprecia que existe una diferencia significativa en la percepción que tienen los chicos y las chicas acerca de la claridad que les aporta la enseñanza tradicional $(p=0,001)$ y la experimental $(p=0,000)$, siendo curiosamente opuestas. Por otro lado, también es significativa la diferencia en la díada Optimismo-Pesimismo $(p=0,044)$ que les genera la enseñanza experimental.

Tabla 4.

Díadas de emociones en función del género. Prueba U de Mann-Whitney.

Enseñanza Tradicional

\begin{tabular}{lcccc}
\hline Díadas & $\begin{array}{c}\text { Seguridad- } \\
\text { Temor }\end{array}$ & $\begin{array}{c}\text { Claridad- } \\
\text { Confusión }\end{array}$ & $\begin{array}{c}\text { Claridad- } \\
\text { Confusión }\end{array}$ & $\begin{array}{c}\text { Optimismo- } \\
\text { Pesimismo }\end{array}$ \\
\hline Z & $-2,035$ & $-3,472$ & $-4,751$ & $-2,015$ \\
\hline Sig. asintótica (bilateral) & 0,042 & 0,001 & 0,000 & 0,044
\end{tabular}

Analizando más pormenorizadamente la figura 9, se pone de manifiesto que las chicas señalaban que la enseñanza tradicional les producía una sensación de mayor claridad y seguridad y que la enseñanza experimental les generaba cierta confusión y pesimismo. Preguntadas posteriormente por esta afirmación, indicaban que, al ser un método de enseñanza nuevo, y dado que todavía no habían recibido la calificación, temían que el hecho de no poder controlar el proceso, puesto que no dependía solo de su estudio, pudiera afectar a sus resultados académicos. Sin embargo, los chicos manifestaron que la enseñanza experimental les resultaba mucho más clara y les producía optimismo y seguridad.

Figura 8.

Díadas de emociones por sexo.

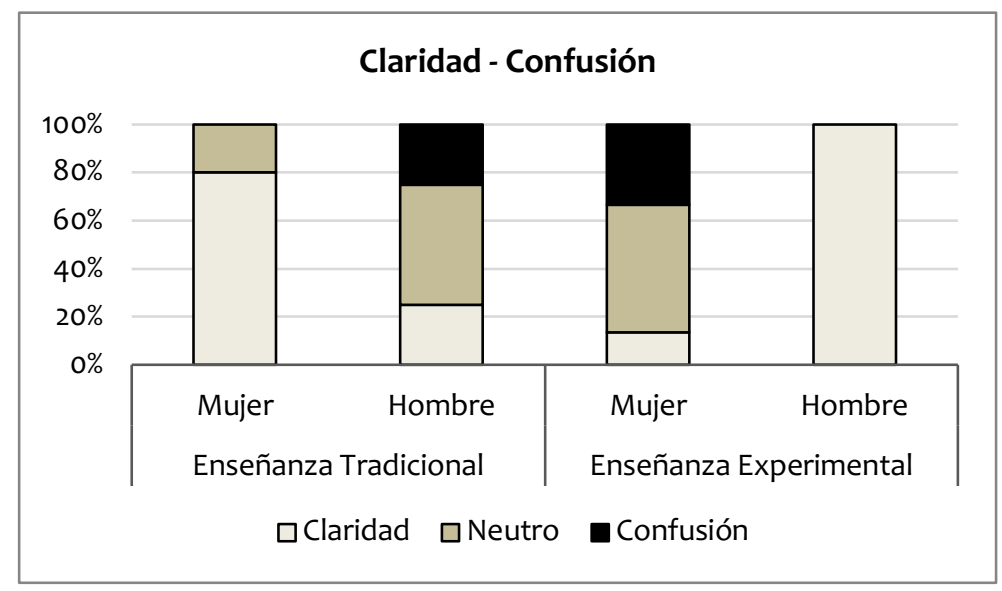



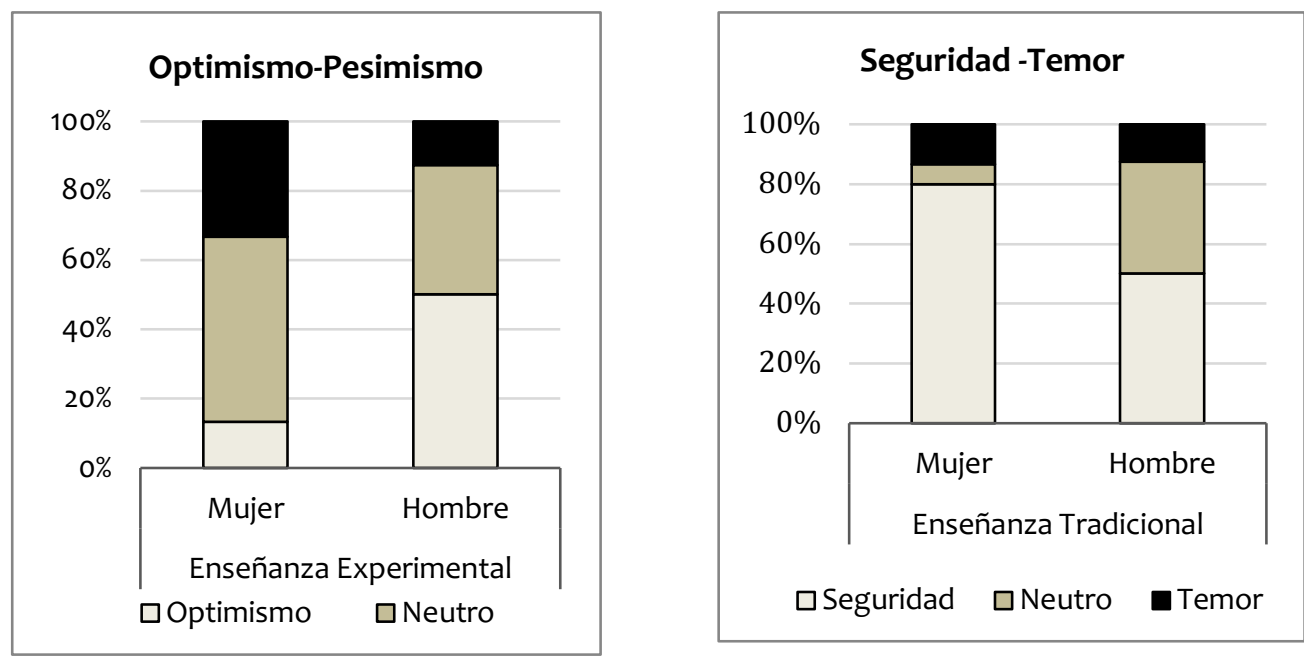

\section{Conclusiones}

La enseñanza y en especial la educación científica se encuentra actualmente ante un gran reto, revertir las actitudes negativas que hacia las materias de ciencias están surgiendo en los estudiantes de los últimos cursos de las enseñanzas previas a la universidad. Se hace pues necesario un nuevo planteamiento que potencie nuevas formas de pensar, enseñar y aprender. Por ello, es preciso una revisión acerca de los modelos sobre la enseñanza y el aprendizaje de las ciencias, de tal modo, que se tengan en cuenta entre otras, las características socioculturales y cognitivas de los alumnos, sus concepciones epistemológicas, pero también sus motivaciones.

Parece lógico pensar, a la luz de lo expresado por los alumnos, que entre las muchas posibles razones que generan un desinterés creciente de los estudiantes hacia el estudio de las ciencias, radica en la percepción que albergan de la poca utilidad que para sus vidas tiene la enseñanza que se les transmite, acrecentado probablemente por la poca interacción que tienen con la adquisición del conocimiento. En este camino encontramos los trabajos de Solbes et al., (2007) realizados con alumnos de bachillerato, que revelan que la enseñanza continúa centrándose en aspectos puramente formales y que no se trabajan aspectos que motivarían al alumnado, como más trabajo de laboratorio o aplicaciones de la Ciencia a la Sociedad, por lo que se continúa ignorando la valoración negativa del alumnado y el abandono especialmente de ciertas materias.

Coincidimos con Campanario, (2004) en que el empleo del artículo científico puede proporcionar al alumnado una oportunidad de tener otro tipo de experiencias científicas, acercándoles a la construcción del conocimiento científico, como parece indicar la buena predisposición del alumnado a la metodología empleada. Así mismo, nos ha permitido observar como tiene otros efectos positivos, pues fomenta la exploración interactiva, promueve el aprendizaje significativo y la colaboración entre los estudiantes. Esto se debe probablemente a que el trabajo grupal genera ambientes cooperativos y favorece la resolución de problemas, que se potencia con el aumento de la motivación derivada de que el alumno se siente consciente de los resultados de su investigación.

Pese a todo, hemos podido comprobar que el alumnado que llega al último curso de secundaria y a bachillerato, incluso procedente de un itinerario científico, raramente ha leído algún artículo de investigación, ni lo ha tratado en ninguna de sus materias de Ciencias. Aun 
así, hemos constatado que a este colectivo le parece muy interesante la utilización del artículo en el aula, por cuanto le ofrece la posibilidad de profundizar en materias que de otra manera le sería imposible estudiar y más aún si es fruto de su propia investigación científica.

Por otro lado, hemos comprobado también que este tipo de propuesta es considerada por el estudiante más amena y creativa que la enseñanza tradicional, a la que consideran monótona y aburrida y que claramente genera en ellos sensaciones positivas, de aprendizaje y de curiosidad, lo cual podría ser un vehículo más que facilite el cambio conceptual tanto cognitivo como afectivo (Mellado et al., 2014).

Así mismo, hemos confirmado que existen diferencias de percepción por parte de chicos y chicas acerca de la enseñanza de la Ciencia. Se ha encontrado que las chicas señalan que la enseñanza tradicional les produce una sensación de mayor claridad y seguridad, frente a una enseñanza experimental que les produce más incertidumbre, fundamentalmente a la hora de obtener buenas calificaciones. Sin embargo, los chicos manifiestan que la enseñanza de las ciencias empleando el artículo de investigación les resulta mucho más clara y les genera actitudes optimistas. Estos datos parecen indicar que efectivamente hay una diferencia en cómo se afronta el estudio de las Ciencias en función del sexo. Este hecho concuerda con estudios anteriores realizados sobre la ansiedad en el estudio de las disciplinas científicas por autores como Udo et al., (2004), entre otros.

Aunque la elaboración de un artículo de investigación se ha mostrado, como un fermento de emociones positivas tanto hacia el estudio de la Ciencia como hacia el proceso científico, implica por parte del docente, un abandono del proceso de enseñanza tradicional y por tanto de su zona de confort, lo que supone un giro de timón en el proceso de enseñanzaaprendizaje y un cambio en su papel, ya que debe pasar del profesor que atesora todo el conocimiento, a ser un guía en el proceso de aprendizaje del alumno.

El presente trabajo, evidencia que se puede ir más allá del binomio explicación-ejercicio tan extendido en nuestras clases de ciencias. Señalándonos cómo el empleo del artículo puede ser una opción didáctica interesante que acerque la ciencia a los intereses y motivaciones del estudiante, generándole experiencias positivas que provoquen la necesaria curiosidad que estimule su capacidad investigadora y, por ende, incite al estudio y aprendizaje de las diferentes disciplinas científicas. En definitiva, esta iniciativa didáctica se postula como un catalizador que fomente las vocaciones científicas en el alumnado.

\section{Referencias}

Bryant, F. B., Kastrup, H., Udo, M., Hislop, N., Shefner, R., \& Mallow, J. (2013). Science Anxiety, Science Attitudes, and Constructivism: A Binational Study. Journal of Science Education and Technology, 22(4), 432-448. https://doi.org/10.1007/s10956-012-9404-x

Buch, T. (2003). CTS desde la perspectiva de la educación tecnológica. Revista Iberoamericana De Educación, 32, 147-163. Revista Iberoamericana de Educación, 32, 147163. https://doi.org/https://doi.org/10.35362/rie320926

Campanario, J. M. (2004). Algunas posibilidades del artículo de investigación como recurso didáctico orientado a cuestionar ideas inadecuadas sobre la ciencia. Enseñanza de Las Ciencias: Revista de Investigación y Experiencias Didácticas, 22(3), 365-378.

Camps, D. (2007). El artículo científico: Desde los inicios de la escritura al IMRYD. Archivos de Medicina, 3(5), 9. http://imed.pub/ojs2/index.php/archmed/article/view/41

Cheung, D. (2009). Students' attitudes toward chemistry lessons: The interaction effect between grade level and gender. Research in Science Education, 39(1), 75-91. https://doi.org/10.1007/s11165-007-9075-4

Edwards, M., Gil, D., Vilches, A., \& Praia, J. (2004). La atención a la situación del mundo en la 
educación científica. Enseñanza de Las Ciencias, 22(1), 47-64.

García-Ruiz, M., \& Sánchez Hernández, B. (2006). Las actitudes relacionadas con las ciencias naturales y sus repercusiones en la práctica docente de profesores de primaria. Perfiles Educativos, XXVIII(114), 61-89.

George, R. (2006). A cross-domain analysis of change in students' attitudes toward science and attitudes about the utility of science. International Journal of Science Education, 28(6), 571-589. https://doi.org/10.1080/09500690500338755

Hargreaves, A. (2000). Mixed emotions: Teachers' perceptions of their interactions with students. Teaching and Teacher Education, 16(8), 811-826. https://doi.org/10.1016/S0742051X(00)00028-7

Mellado, V., Borrachero, A. B., Brígido, M., Melo, L. V., Dávila, M. A., Cañada, F., Conde, M. C., Costillo, E., Cubero, J., Esteban, R., Martínez, G., Ruiz, C., Sánchez, J., Garritz, A., Mellado, L., Vázquez, B., Jiménez, R., \& Bermejo, M. L. (2014). Emotions in science teaching. Ensenanza de Las Ciencias, 32(3), 11-36. https://doi.org/10.5565/rev/ensciencias.1478

Mora Teruel, F. (2017). Neuroeducación. Solo Se Puede Aprender Aquello Que Se Ama. (2 edición). Alianza Editorial.

Murillo, F. J., Martínez-Garrido, C., \& Belavi, G. (2017). Sugerencias para Escribir un Buen Artículo Científico en Educación. REICE. Revista Iberoamericana Sobre Calidad, Eficacia y Cambio En Educación, 15(3), 5-34. https://doi.org/10.15366/reice2017.15.3.001

Pelcastre Villafuerte, L., Gómez Serrato, A. R., \& Zavala, G. (2015). Actitudes hacia la ciencia de estudiantes de educación preuniversitaria del centro de México. Revista Eureka Sobre Enseñanza y Divulgación de Las Ciencias., 12(3), 475-490. https://doi.org/10.25267/rev_eureka_ensen_divulg_cienc.2015.v12.i3.06

Pérez, A., \& De Pro, A. (2013). Estudio demoscópico de lo que sienten y piensan los niños y adolescentes sobre la enseñanza formal de las ciencias. En V. Mellado, L.J. Blanco, A.B. Borrachero y J.A. Cárdenas (Eds.),. In Las Emociones en la Enseñanza y el Aprendizaje de las Ciencias y las Matemáticas. Badajoz: DEPROFE.

Ramos-Álvarez, M. M., \& Catena, A. (2004). Normas para la elaboración y revisión de artículos originales experimentales en Ciencias del Comportamiento. International Journal of Clinical and Health Psychology, 4(1), 173-189.

Schreiner, C., \& Sjøberg, S. (2004). Sowing the seeds of rose: Background, rationale, questionnaire development and data collection for ROSE (The Relevance of Science Education) - a comparative study of students' views of science and science education. In Acta Didactica (p. 126).

Slafer, G. A. (2009). ¿Como escribir un artículo científico? Revista de Investigación En Educación, 6, 124-132.

Solbes, J., Montserrat, R., \& Furió, C. (2007). El desinterés del alumnado hacia el aprendizaje de la ciencia: implicaciones en su enseñanza. Didáctica De Las Ciencias Experimentales $Y$ Sociales, 117, 91-117.

Udo, M. K., Ramsey, G. P., \& Mallow, J. V. (2004). Science Anxiety and Gender in Students Taking General Education Science Courses. Journal of Science Education and Technology, 13(4), 435-446. https://doi.org/10.1007/s10956-004-1465-z

Vázquez, Á., \& Manassero, M. A. (2008). El declive de las actitudes hacia la ciencia de los estudiantes: un indicador inquietante para la educación científica. Revista Eureka Sobre Enseñanza y Divulgación de Las Ciencias., 8(3), 274-292. https://doi.org/10.25267/rev_eureka_ensen_divulg_cienc.2008.v5.i3.03 